\title{
Reservoir biteguard improves quality of life of people with xerostomia
}

\author{
What is the effect of a reservoir biteguard for artificial saliva on the oral health- \\ related quality of life of people who have xerostomia?
}

\section{Robinson PG, Pankhurst CL, Garrett EJ. Randomized-controlled trial: effect of a reservoir biteguard on quality of life in xerostomia. J Oral Pathol Med 2005; 34:193-197}

Design A double-blind randomised controlled trial was conducted in two London teaching hospitals.

Intervention Patients who had symptomatic xerostomia were randomised into either the study (reservoir biteguard) or the control (conventional biteguard) groups. All participants were asked to wear the biteguards at night-time and at other times during the day if they preferred. They were provided with aqueous gel and artificial saliva spray for symptomatic relief of their xerostomia for the duration of the study.

Outcome measure Outcomes were number of impacts and total scores as recorded by a modified version of oral impacts on daily performance (OIDP). They were measured at baseline and 1 year after wearing a biteguard.

Results Of 136 people who were invited to participate, 92 gave informed consent but six declined to participate before randomisation. At 1-month followup, 84 people remained in the trial. The median number of impacts in the study and control groups was three and four respectively. The median total score was six and 12 respectively. In ANCOVA, receipt of the reservoir biteguard reduced the number of impacts recorded by OIDP but there was no significant difference in the total score.

Conclusions Reservoir biteguards improved the quality of life of people with xerostomia by reducing the number of impacts on daily life.
Address for correspondence: Professor Peter G Robinson, Department of Oral Health and Development, School of Clinical Dentistry, Claremont Crescent, Sheffield S10 2TA, UK. E-mail: peter.g.robinson@sheffield.ac.uk.

\section{Commentary}

Recent research has suggested a relationship between chronic dry mouth and oral-health-related quality of life (OHRQoL) among older adults. ${ }^{1}$ Dry mouth affects important aspects of life such as speaking, the enjoyment and eating of food, and the wearing of dental prostheses.

Professor Robinson and colleagues have undertaken an interesting and ambitious project which aimed to determine whether using a 'reservoir biteguard' to deliver a saliva substitute to the oral cavity improves sufferers' OHRQoL. The choice of the RCT design was appropriate, for the researchers set out to determine the intervention's efficacy. Participants were randomly allocated to either a test or a control group, and a comparison of their baseline characteristics indicated that the randomisation had worked. The intervention itself was a reservoir splint worn over the lower teeth at night (and during the day if preferred). Subjects in the control group wore a splint which was similar but did not have a reservoir. The intervention description in the Methods section ("All participants were provided with aqueous gel... and artificial saliva spray... for symptomatic relief of their xerostomia for the duration of the study") does not explicitly state what was placed in the reservoir, and the reader can only assume that artificial saliva was indeed placed in it, and that the aforementioned spray and the gel were used as well as the intervention. At 93\%, the follow-up rate was acceptable.

The outcome measure was an established OHRQoL measure, and no substantial difference was found between the two groups after 4 weeks. This suggests that using a reservoir biteguard offers no detectable improvement in OHRQoL in xerostomia sufferers. It would have been useful to first determine whether the intervention did alleviate participants' dry-mouth symptoms; after all, even if a measurable difference in OHRQoL between the two groups had been observed, the next question to be answered would have been whether it was in fact due to intervention-associated differences between the two groups in their experience of xerostomia. Thus, data on the occurrence of dry mouth in the two groups (before and after the intervention) would have been helpful, as would some information on the discretionary use of the aqueous gel and saliva spray by each group: it is possible that the null finding could be accounted for by greater use of those adjuncts by the control group.

\section{Practice point}

- This study suggests that reservoir biteguards are not useful in improving xerostomia sufferers' oral-health-related quality of life. Further investigation of the biteguards' ability to alleviate the symptoms of dry mouth is warranted, however.

\section{Murray Thomson}

Department of Oral Sciences, School of Dentistry, The University of Otago, Dunedin, New Zealand.

1. Locker D. Dental status, xerostomia and the oral health-related quality of life of an elderly institutionalized population. Spec Care Dent 2003; 23: 86-93. 


\section{Professor Robinson responds on behalf of the authors:}

We thank Dr Thompson for his kind and thoughtful commentary on our paper. He raises many good points and we welcome the opportunity to comment on them. I would like to point out that we did collect many of the data to which Dr Thompson refers, which were omitted from the publication due to space.

While the intervention is not described explicitly in the methods section it is described in the introduction, 'The reservoirs are filled using a monojet syringe, and the substitute saliva leaks through the holes to bathe the cheeks and through the inner slit to the teeth and gums'.

Both the control and the test groups were provided with their supplies of artificial saliva and except for specific instructions on how to fill the reservoir were given the same instructions on how and when to use the lubricants. The emphasis is on reservoir and not the biteguard. The splint reservoir provides a slow release of artificial saliva. The use of the placebo splint in the controls was to control out any mechanical stimulation of salivary flow. Artificial salivas are well recognised to provide symptomatic relief in users.

While we would agree there is no significant difference for the total score, table 3 in the original paper, shows that people using the reservoir biteguards had fewer impacts than the controls.

Prior to receiving a research grant to conduct this study we had evaluated this slow release device for about one year and it had been well received by patients. As a measurable difference in OHRQoL between the two groups was observed, we concluded that this dif- ference was due to the intervention. However, we acknowledge that prior longitudinal data for OIDP associated with the device would have helped to interpret the findings.

All the participants had dry mouth before and after the study (ie they had salivary flow levels $<0.2 \mathrm{ml} / \mathrm{min}$ ) but the levels of symptoms were also similar using Dr Murray's Xerostomia inventory (XI $)^{1}$. The mean XI (SE) in the control and study groups were 34.6 (1.6) and 35.4 (1.6) respectively before the intervention and 32.7 (1.7) and 32.1(1.8) afterwards. Before the study artificial saliva was used by $12.8 \%$ of the controls and $7.0 \%$ in study group. We do not have data for use of the artificial saliva (on its own) after the study but we have data on the helpfulness of this treatment. The mean ranking of this treatment in the control group was 3.1 and in the study group it was 3.2. Thus we do not believe the use of artificial saliva on its own affected the results.

In conclusion, we can see that some of the questions raised by $\mathrm{Dr}$ Thompson arise from the requirements of the journal for papers that are as brief as possible. Moreover, we agree entirely that more work is required, not only on the subjective experience of xerostomia and treatments for it, but also on the relationships between impairments (such as reduced salivary flow), symptoms (for example, as measured by the XI) and oral health-related quality of life.

1. Thomson WM, Chalmers JM, Spencer AJ, Williams SM. The Xerostomia Inventory: a multi-item approach to measuring dry mouth. Community Dent Health. 1999 Mar;16(1):12-7.

Evidence-Based Dentistry (2006) 7, 42-43.

doi:10.1038/sj.ebd.6400392 